Georgian Mathematical Journal

Volume 8 (2001), Number 1, 33-59

\title{
WEIGHT INEQUALITIES FOR SINGULAR INTEGRALS DEFINED ON SPACES OF HOMOGENEOUS AND NONHOMOGENEOUS TYPE
}

\author{
D. E. EDMUNDS, V. KOKILASHVILI, AND A. MESKHI
}

Dedicated to Professor Hans Triebel on his 65th birthday

\begin{abstract}
Optimal sufficient conditions are found in weighted Lorentz spaces for weight functions which provide the boundedness of the CalderónZygmund singular integral operator defined on spaces of homogeneous and nonhomogeneous type.
\end{abstract}

2000 Mathematics Subject Classification: 42B20, 42B25.

Key words and phrases: Singular Integral, maximal function, Hardytype operator, space of homogeneous type, space of nonhomogeneous type, Lorentz space.

\section{INTRODUCTION}

In the present paper optimal sufficient conditions are found for the weight functions which provide the boundedness of Calderón-Zygmund singular integral operator defined on spaces of homogeneous as well as nonhomogeneous type in weighted Lorentz spaces. In the nonhomogeneous case the results are new even in Lebesgue spaces.

Two-weight strong type inequalities for singular integrals in Lebesgue spaces on spaces of homogeneous type (SHT) were established in [5] (see also [6], Chapter 9, and [14]), while a similar problem for singular integrals defined on homogeneous groups was considered in [16] in the case of Lorentz spaces.

Two-weight inequalities for Hilbert transforms with monotonic weights were studied in [17]. Analogous problems for singular integrals in Euclidean spaces were considered in [8] and generalized in [7] for singular integrals on Heisenberg groups. Optimal conditions for a pair of weights ensuring the validity of twoweight inequalities for Calderón-Zygmund singular integrals were obtained in [3] (and also in [19] for the case of Lorentz spaces). The latter result was generalized to the setting of homogeneous groups in [13], [15].

Finally, we would like to mention that singular integrals on spaces of nonhomogeneous type (measure spaces with a metric) were studied in [18].

ISSN 1072-947X / \$8.00 / (c) Heldermann Verlag www.heldermann.de 


\section{Preliminaries}

In this section we give the notion of SHT and defined Lorentz space. The definition of a singular integral and several well-known results concerning the Hardy-type operators on SHT are also given.

Definition 1.1. A space of homogeneous type (SHT) $(X, d, \mu)$ is a topological space $X$ endowed with a complete measure $\mu$ such that: $(a)$ the space of compactly supported continuous functions is dense in $L^{1}(X, \mu)$, and $(b)$ there exists a non-negative real-valued function (quasimetric) $d: X \times X \rightarrow \mathbb{R}^{1}$ satisfying:

(i) $d(x, x)=0$ for arbitrary $x \in X$;

(ii) $d(x, y)>0$ for arbitrary $x, y \in X, x \neq y$;

(iii) there exists a positive constant $a_{0}$ such that the inequality

$$
d(x, y) \leq a_{0} d(y, x)
$$

holds for all $x, y \in X$;

(iv) there exists a positive constant $a_{1}$ such that the inequality

$$
d(x, y) \leq a_{1}(d(x, z)+d(z, y))
$$

holds for arbitrary $x, y, z \in X$;

(v) for every neighborhood $V$ of any point $x \in X$ there exists a number $r>0$ such that the ball

$$
B(x, r)=\{y \in X: d(x, y)<r\}
$$

with center in $x$ and radius $r$ is contained in $V$;

(vi) the balls $B(x, r)$ are measurable for all $x \in X, r>0$ and, moreover, $0<\mu B(x, r)<\infty$;

(vii) there exists a positive constant $b$ such that the inequality (doubling condition)

$$
\mu B(x, 2 r) \leq b \mu B(x, r)
$$

is true for all $x \in X$ and for all positive $r$.

It will be supposed that for some $x_{0} \in X$

$$
\mu\left\{x_{0}\right\}=\mu\left\{x \in X: d\left(x_{0}, x\right)=a\right\}=0,
$$

where

$$
a \equiv \sup \left\{d\left(x_{0}, x\right): \quad x \in X\right\} .
$$

Note that the condition $a=\infty$ is equivalent to the condition $\mu(X)=\infty$, and if $a=\infty$, then $\mu\left\{x \in X: d\left(x_{0}, x\right)=a\right\}=0$.

For the definitions, various examples and properties of SHT, we refer to [2], [23] (also to [6]).

Definition 1.2. By a spaces of nonhomogeneous type we mean a measure space with a quasimetric $(X, d, \mu)$ satisfying conditions (i)-(v) of Definition 1.1, i.e., the doubling condition is not assumed and may fail. 
In the sequel it will be assumed that there exists a point $x_{0} \in X$ such that

$$
B\left(x_{0}, R\right) \backslash B\left(x_{0}, r\right) \neq \varnothing
$$

for all $r$ and $R$ provided $0<r<R<a$.

In the remaining part of this section $(X, d, \mu)$ is assumed to be a space of nonhomogeneous type.

Definition 1.3. An almost everywhere positive, locally integrable function $w: X \rightarrow \mathbb{R}^{1}$ is called a weight.

For weight functions $w$, we denote by $L_{w}^{p q}(X)$ the Lorentz space with weight $w$ which represents the class of all $\mu$-measurable functions $f: X \rightarrow \mathbb{R}^{1}$ for which

$$
\|f\|_{L_{w}^{p q}(X)}=\left(q \int_{0}^{+\infty}\left(\int_{\{x \in X:|f(x)|>\lambda\}} w(x) d \mu\right)^{q / p} \lambda^{q-1} d \lambda\right)^{1 / q}<\infty
$$

when $1 \leq p \leq \infty, 1 \leq q<\infty$, and

$$
\|f\|_{L_{w}^{p \infty}(X)}=\sup _{\lambda>0} \lambda\left(\int_{\{x \in X:|f(x)|>\lambda\}} w(x) d \mu\right)^{1 / p}<\infty
$$

when $1<p<\infty$.

It is clear that if $p=q$, then $L_{w}^{p q}(X)=L_{w}^{p}(X)$ is a Lebesgue space. If $w \equiv 1$, then we put

$$
L_{w}^{p}(X) \equiv L^{p}(X) .
$$

The following lemma holds.

Lemma A. Let $E \subset X$ be a $\mu$-measurable set, $1<p, q<\infty$ and suppose that $w$ is a weight function on $X$. Further, let $f, f_{1}$ and $f_{2}$ be $\mu$-measurable functions on $X$. Then:

(i)

$$
\left\|\chi_{E}(\cdot)\right\|_{L_{w}^{p q}(X)}=\left(\int_{E} w(x) d \mu\right)^{1 / p}
$$

$$
\|f\|_{L_{w}^{p q_{1}}(X)} \leq\|f\|_{L_{w}^{p q_{2}(X)}}
$$

for fixed $p$ and $q_{1} \leq q_{2}$;

(iii) there exists a positive constant $c$ such that

$$
\left\|f_{1} f_{2}\right\|_{L_{w}^{p q}(X)} \leq c\left\|f_{1}\right\|_{L_{w}^{p_{1} q_{1}}(X)}\left\|f_{2}\right\|_{L_{w}^{p_{2} q_{2}}(X)},
$$

where $\frac{1}{p}=\frac{1}{p_{1}}+\frac{1}{p_{2}}, \frac{1}{q}=\frac{1}{q_{1}}+\frac{1}{q_{2}}$.

The first part of this Lemma is proved in [22], Chpater V, Section 3 and the rest of the proof is given in [10].

Lemma B ([1], [19]). Let $\left\{E_{k}\right\}$ be a sequence of $\mu$-measurable subsets of $X$, such that $\sum_{k} \chi_{E_{k}} \leq c_{0} \chi_{\cup E_{k}}$. Then 
(i) there exists a positive constant $k_{1}=k_{1}\left(c_{0}\right)$, such that for arbitrary $f$, the following inequality holds:

$$
\sum_{k}\left\|f(\cdot) \chi_{E_{k}(\cdot)}\right\|_{L_{w}^{r s}(X)}^{\lambda} \leq k_{1}\left\|f(\cdot) \chi_{\cup E_{k}(\cdot)}\right\|_{L_{w}^{r s}(X)}^{\lambda},
$$

where $\max \{r, s\} \leq \lambda$;

(ii) there exists a positive constant $k_{2}=k_{2}\left(c_{0}\right)$, such that for every $f$, the following inequality holds:

$$
\left\|f(\cdot) \chi_{\cup E_{k}(\cdot)}\right\|_{L_{w}^{p q}(X)}^{\gamma} \leq k_{2} \sum_{k}\left\|f(\cdot) \chi_{E_{k}(\cdot)}\right\|_{L_{w}^{p q}(X)}^{\gamma},
$$

where $0<\gamma \leq \min \{p, q\}$.

Definition 1.4. If $1<p<\infty$, then $A_{p}(X)$ is the set of all weights $w$ such that

$$
\sup \left(\frac{1}{\mu(B)} \int_{B} w(x) d \mu\right)\left(\frac{1}{\mu(B)} \int_{B} w^{1-p^{\prime}}(x) d \mu\right)^{p-1}<\infty, \quad p^{\prime}=\frac{p}{p-1},
$$

where the supremum is taken over all balls $B \subset X$.

We recall that $a \equiv \sup \left\{d\left(x_{0}, x\right): x \in X\right\}$ for some $x_{0} \in X$ with $\mu\left\{x_{0}\right\}=0$.

Lemma $\mathbf{C}([5])$. Let $a=\infty, 1<p<\infty, \rho \in A_{p}(X)$ and $0<c_{1} \leq c_{2}<$ $c_{3}<\infty$. Then there exists a positive constant $c$ such that the inequality

$$
\int_{B\left(x_{0}, c_{3} t\right) \backslash B\left(x_{0}, c_{2} t\right)} \rho(x) d \mu \leq c \int_{B\left(x_{0}, c_{1} t\right)} \rho(x) d \mu
$$

holds for arbtrary $t>0$.

In the sequel we shall need the following results concerning the Hardy-type operators on the measure space $(X, \mu)$

$$
\mathcal{H} f(t)=\int_{\left\{x: d\left(x_{0}, x\right)<t\right\}} f(x) d \mu(x), \quad t \in(0, a),
$$

and

$$
\widetilde{\mathcal{H}} f(t)=\int_{\left\{x: d\left(x_{0}, x\right)>t\right\}} f(x) d \mu(x), \quad t \in(0, a) .
$$

The following result is from [5].

Proposition A. Let $1<p \leq q<\infty, \mu\left\{x_{0}\right\}=0$. Then the inequality

$$
\left(\int_{0}^{a} v(t)|\mathcal{H} f(t)|^{q} d t\right)^{1 / q} \leq c\left(\int_{X}|f(x)|^{p} w(x) d \mu\right)^{1 / p},
$$

holds with some $c>0$ independent of $f, f \in L_{w}^{p}(X)$, if and only if

$$
D=\sup _{0<t<a}\left(\int_{t}^{a} v(\tau) d \tau\right)^{1 / q}\left(\int_{\left\{x: d\left(x_{0}, x\right)<t\right\}} w^{1-p^{\prime}}(x) d \mu\right)^{1 / p^{\prime}}<\infty .
$$


Moreover, if $c$ is the best constant in (1.2), then $D \leq c \leq 4 D$.

Proposition B ([5]). Let $1<p \leq q<\infty, \mu\left\{x: d\left(x_{0}, x\right)=a\right\}=0$. Then the inequality

$$
\left(\int_{0}^{a} v(t)|\widetilde{\mathcal{H}} f(t)|^{q} d t\right)^{1 / q} \leq c\left(\int_{X}|f(x)|^{p} w(x) d \mu\right)^{1 / p}
$$

holds with a constant $c$ independent of $f, f \in L_{w}^{p}(X)$, if and only if

$$
\widetilde{D}=\sup _{0<t<a}\left(\int_{0}^{t} v(\tau) d \tau\right)^{1 / q}\left(\int_{\left\{x: d\left(x_{0}, x\right)>t\right\}} w^{1-p^{\prime}}(x) d \mu\right)^{1 / p^{\prime}}<\infty .
$$

Moreover, if $c$ is the best constant in (1.3), then $\widetilde{D} \leq c \leq 4 \widetilde{D_{1}}$.

Let $f$ be a $\mu$-measurable function defined on $X$ and suppose that

$$
H_{\varphi \psi} f(x)=\varphi(x) \int_{B\left(x_{0}, d\left(x_{0}, x\right)\right)} f(y) \psi(y) w(y) d \mu(y)
$$

is a Hardy-type operator on $X$, where $\varphi$ and $\psi$ are positive $\mu$-measurable functions on $X$.

The following proposition is true (see Theorem 2.4 of [4] for $a=\infty$. The case $a<\infty$ can be obtained similarly).

Proposition C. Let $r=s=1$ or $r \in(0, \infty]$ and $s \in[1, \infty], p=q=1$ or $p \in(1, \infty), q \in[1, \infty], \max \{r, s\} \leq \min \{p, q\}$. The operator $H_{\varphi \psi}$ is bounded from $L_{w}^{r s}(X)$ to $L_{v}^{p q}(X)$ if and only if

$$
A \equiv \sup _{0<t<a}\left\|\varphi(\cdot) \chi_{\left\{d\left(x_{0}, y\right)>t\right\}}(\cdot)\right\|_{L_{v}^{p q}(X)}\left\|\psi(\cdot) \chi_{\left\{d\left(x_{0}, y\right) \leq t\right\}}(\cdot)\right\|_{L_{w}^{r^{\prime} s^{\prime}}(X)}<\infty
$$

with $\left\|H_{\varphi \psi}\right\| \approx A$.

Let

$$
H_{\varphi \psi}^{\prime} f(x)=\varphi(x) \int_{\left\{y: d\left(x_{0}, y\right)>d\left(x_{0}, x\right)\right\}} f(y) \psi(y) d \mu(y) .
$$

By the dual arguments we deduce the following result:

Proposition D. Let $r, s, p$ and $q$ satisfy the conditions of Proposition C. The operator $H_{\varphi, \psi}^{\prime}$ is bounded from $L_{w}^{r s}(X)$ to $L_{v}^{p q}(X)$ if and only if:

$$
A^{\prime}=\sup _{t>0}\left\|\varphi(\cdot) \chi_{\left\{d\left(x_{0}, y\right) \leq t\right\}}(\cdot)\right\|_{L_{v}^{p q}(X)}\left\|\psi(\cdot) \chi_{\left\{d\left(x_{0}, y\right)>t\right\}}(\cdot)\right\|_{L_{w}^{r^{\prime} s^{\prime}}(X)}<\infty
$$

with $\left\|H_{\varphi \psi}^{\prime}\right\| \approx A^{\prime}$. 


\section{Singular Integrals ON SHT}

In this section the existence of singular integrals is investigated and optimal sufficient conditions for the boundedness of singular integral operators in weighted Lorentz spaces defined on $\operatorname{SHT}(X, d, \mu)$ are established.

Let $k: X \times X \backslash\{(x, x): x \in X\} \rightarrow \mathbb{R}^{1}$ be a measurable function satisfying the condition

$$
|k(x, y)| \leq \frac{c}{\mu B(x, d(x, y))}
$$

for all $x, y \in X, x \neq y$, and

$$
\left|k\left(x_{1}, y\right)-k\left(x_{2}, y\right)\right|+\left|k\left(y, x_{1}\right)-k\left(y, x_{2}\right)\right| \leq c \omega\left(\frac{d\left(x_{2}, x_{1}\right)}{d\left(x_{2}, y\right)}\right) \frac{1}{\mu\left(B\left(x_{2}, d\left(x_{2}, y\right)\right)\right)},
$$

for all $x_{1}, x_{2}, y \in X$ provided that $d\left(x_{2}, y\right)>b d\left(x_{1}, x_{2}\right)$, where $\omega$ is a positive non-decreasing function on $(0, \infty)$ satisfying the $\Delta_{2}$-condition (i.e., $\omega(2 t) \leq$ $c \omega(t)$ for all $t \in(0, \infty))$ and the Dini condition

$$
\int_{0}^{1} \frac{\omega(t)}{t} d t<\infty
$$
limit

We shall also suppose that for some $p_{0}, 1<p_{0}<\infty$, and all $f \in L^{p_{0}}(X)$, the

$$
K f(x)=\lim _{\varepsilon \rightarrow 0+} \int_{X \backslash B(x, \varepsilon)} k(x, y) f(y) d \mu
$$

exists a.e. on $X$ and that the operator $K$ is bounded in $L_{w}^{p}(X)$.

For the definition of singular integrals and for other related remarks see, e.g., [21], Chapter I, pp. 29-36, also [6], p. 295, and [5].

Theorem A ([9]). Let $1<p<\infty$. If $w \in A_{p}(X)$, then the operator $K$ is bounded in $L_{w}^{p}(X)$.

Theorem B ([12], p. 207). Let $1<p, q<\infty$. If $w \in A_{p}(X)$, then the operator $K$ is bounded in $L_{w}^{p q}(X)$. If the Hilbert transform $H$,

$$
H f(x)=p \cdot v \cdot \int_{-\infty}^{+\infty} f(t)(x-t)^{-1} d t
$$

is bounded in $L_{w}^{p q}(\mathbb{R})$, then $w \in A_{p}(\mathbb{R})$.

The following Lemma holds.

Lemma 2.1. Let $1<s \leq p<\infty, \rho \in A_{p}(X)$. Suppose that for weight functions $w$ and $w_{1}$ the following conditions hold:

(1) there exist a positive increasing function $\sigma$ defined on $\left(0,4 a_{1} a\right)$ and a positive constant $b$ such that

$$
\sigma\left(d\left(x_{0}, x\right)\right) \rho(x) \leq b w(x) w_{1}(x)
$$

for almost all $x \in X$; 
(2) for all $t, 0<t<a$, we have

$$
\left\|\frac{1}{w(\cdot) w_{1}(\cdot)} \chi_{\left\{d\left(x_{0}, y\right) \leq t\right\}}(\cdot)\right\|_{L_{w}^{p^{\prime} s^{\prime}}(X)}<\infty .
$$

Then $K g(x)$ exists a.e. on $X$ for any $g$ satisfying the condition

$$
\left\|g(\cdot) w_{1}(\cdot)\right\|_{L_{w}^{p s}(X)}<\infty .
$$

Proof. Let us fix some positive $\alpha$ satisfying $0<\alpha<\frac{\alpha}{\alpha_{1}}$ and put

$$
S_{\alpha}=\left\{x \in X: \quad d\left(x_{0}, x\right) \geq \frac{\alpha}{2}\right\} .
$$

Assume that $\left\|g(\cdot) w_{1}(\cdot)\right\|_{L_{w}^{p s}(X)}<\infty$. Introduce g in the following way:

$$
g(x)=g_{1}(x)+g_{2}(x),
$$

where $g_{1}(x)=g(x) \cdot \chi_{S_{a}}(x), g_{2}(x)=g(x)-g_{1}(x)$.

For $g_{1}$ we have

$$
\begin{gathered}
\int_{X}\left|g_{1}(x)\right|^{p} \rho(x) d \mu=\frac{\sigma(\alpha / 2)}{\sigma(\alpha / 2)} \int_{S_{a}}|g(x)|^{p} \rho(x) d \mu \\
\leq \frac{1}{\sigma(\alpha / 2)} \int_{S_{a}}|g(x)|^{p} \rho(x) \sigma\left(2 a_{1} d\left(x_{0}, x\right)\right) d \mu \leq \frac{b}{\sigma(\alpha / 2)} \int_{S_{a}}|g(x)|^{p} w_{1}^{p}(X) w(x) d \mu \\
=\frac{b_{1}}{\sigma(\alpha / 2)}\left\|g(\cdot) w_{1}(\cdot)\right\|_{L_{w}^{p}(X)}^{p} \leq \frac{b}{\sigma(\alpha / 2)}\left\|g(\cdot) w_{1}(\cdot)\right\|_{L_{w}^{p s}(X)}^{p} .
\end{gathered}
$$

(In the latter inequality we have used Lemma B, part (ii).) Hence $g_{1} \in L_{\rho}^{p}(X)$ and, according to Theorem A, $K g \in L_{\rho}^{p}(X)$ and consequently $K g(x)$ exists a.e. on $X$.

Now, let $d\left(x_{0}, x\right)>\alpha a_{1}$, and let $d\left(x_{0}, y\right)<\alpha / 2$. We have

$$
d\left(x_{0}, x\right) \leq a_{1}\left(d\left(x_{0}, y\right)+d(y, x)\right) \leq a_{1}\left(d\left(x_{0}, y\right)+a_{0} d(x, y)\right) .
$$

Hence

$$
d(x, y) \geq \frac{d\left(x_{0}, x\right)}{a_{1} a_{0}}-\frac{1}{a_{0}} d\left(x_{0}, y\right) \geq \frac{\alpha}{a_{0}}-\frac{\alpha}{2 a_{0}}=\frac{\alpha}{2 a_{0}} .
$$

Moreover,

$$
B\left(x_{0}, d(x, y)\right) \subset B\left(x, a_{1}\left(1+a_{1}\left(1+a_{0}^{2}\right)\right) d(x, y)\right)
$$

and consequently we obtain the inequality

$$
\mu B\left(x_{0}, \alpha / 2\right) \leq c_{1} \mu B(x, d(x, y))
$$


where $c_{1}$ is independent of $x$. So, for $K g_{2}$, using the latter estimate and the Hölder's inequality, we have

$$
\begin{gathered}
\left|K g_{2}(x)\right|=\left|\int_{X} g(y) k(x, y) d \mu\right| \leq c_{2} \int_{B\left(x_{0}, \alpha / 2\right)} \frac{|g(y)|}{\mu B(x, d(x, y))} d \mu \\
\leq \frac{c_{3}}{\mu B\left(x_{0}, \frac{\alpha}{2 c_{0}}\right)} \int_{B\left(x_{0}, \alpha / 2\right)}|g(y)| d \mu \\
\leq \frac{c_{3}}{\mu B\left(x_{0}, \frac{\alpha}{2 c_{0}}\right)} \int_{B\left(x_{0}, \alpha / 2\right)}|g(y)| \frac{1}{w(y) w_{1}(y)} w_{1}(y) w(y) d \mu \\
\leq \frac{c_{3}}{\mu B\left(x_{0}, \frac{\alpha}{2 c_{0}}\right)}\left\|\chi_{B\left(x_{0}, \frac{\alpha}{2}\right)}(\cdot) \frac{1}{w(\cdot) w_{1}(\cdot)}\right\|_{L_{w}^{p^{\prime} s^{\prime}(X)}}\left\|g(\cdot) w_{1}(\cdot)\right\|_{L_{w}^{p s}(X)}<\infty .
\end{gathered}
$$

Thus $K g_{2}(x)$ converges absolutely for all $x$ with $d\left(x_{0}, x\right)>\alpha a_{1}$. Since we can choose $\alpha$ arbitrarily small and $\mu\left\{x_{0}\right\}=0$, we conclude that $K g(x)$ exists $\mu$-a.e. on $X$.

From Lemma 2.1 it is easy to derive

Lemma 2.2. Let $1<s \leq p<\infty$. If $u$ and $u_{1}$ are positive increasing functions on $\left(0,4 a_{1} a\right)$ and

$$
\left\|\frac{1}{u\left(d\left(x_{0}, \cdot\right)\right) u_{1}\left(d\left(x_{0}, \cdot\right)\right)} \chi_{\left\{d\left(x_{0}, y\right) \leq t\right\}}(\cdot)\right\|_{L_{u\left(d\left(x_{0}, \cdot\right)\right)}^{p^{\prime} s^{\prime}}(X)}<\infty
$$

for all $t$ satisfying $0<t<a$, then for arbitrary $g$ satisfying the condition

$$
\left\|\varphi(\cdot) u_{1}\left(d\left(x_{0}, \cdot\right)\right)\right\|_{L_{u\left(d\left(x_{0}, \cdot\right)\right)}^{p s}(X)}<\infty
$$

$K \varphi(x)$ exists a.e. on $X$.

The following lemma is proved in the same way as Lemma 2.1.

Lemma 2.3. Let $a=\infty, 1<s \leq p<\infty, \rho \in A_{p}(X)$. If the weight functions $w$ and $w_{1}$ satisfy the conditions:

(1) there exists a decreasing positive function $\sigma$ on $(0, \infty)$ such that for almost all $x \in X$ we have

$$
\sigma\left(d\left(x_{0}, \cdot\right)\right) \rho(x) \leq b w(x) w_{1}^{p}(x),
$$

where the positive constant $b$ does not depend on $x \in X$;

(2) if for every $t>0$

$$
\left\|\frac{\mu B\left(d\left(x_{0}, \cdot\right)\right)^{-1}}{w(\cdot) w_{1}(\cdot)} \chi_{\left\{d\left(x_{0}, y\right)>t\right\}}(\cdot)\right\|_{L_{w}^{p^{\prime} s^{\prime}}(X)}<\infty,
$$

then $K g(x)$ exists a.e. on $X$ for arbitrary $g$ provided that $\left\|g(\cdot) w_{1}(\cdot)\right\|_{L_{w}^{p s}(X)}<\infty$.

From Lemma 2.3 the next lemma follows easily: 
Lemma 2.4. Let $a=\infty, 1<s \leq p<\infty$. Assume that $u$ and $u_{1}$ are positive decreasing functions on $(0, \infty)$ and the condition

$$
\left\|\frac{\mu B\left(d\left(x_{0}, \cdot\right)\right)^{-1}}{u\left(d\left(x_{0}, \cdot\right)\right) u_{1}\left(d\left(x_{0}, \cdot\right)\right)} \chi_{\left\{d\left(x_{0}, y\right)>t\right\}}(\cdot)\right\|_{L_{u\left(d\left(x_{0}, \cdot\right)\right.}^{p^{\prime} s^{\prime}}(X)}<\infty
$$

holds for any $t>0$. Then $K g(x)$ exists a.e. on $X$ for arbitrary $g$ satisfying the condition $g(\cdot) u_{1}\left(d\left(x_{0}, \cdot\right)\right) \in L_{u\left(d\left(x_{0}, \cdot\right)\right)}^{p s}(X)$.

Now we pass to the weight inequalities for the operator $K$.

Theorem 2.1. Let $1<s \leq p \leq q<\infty$, let $w$ be a weight function on $X$, suppose that $\sigma$ is a positive increasing continuous function on $\left(0,4 a_{1} a\right)$, $\rho \in A_{p}(X)$ and $v(x)=\sigma\left(d\left(x_{0}, x\right)\right) \rho(x)$. Suppose the following conditions are satisfied:

(a) there exists a positive constant b such that the inequality

$$
\sigma\left(2 a_{1} d\left(x_{0}, x\right)\right) \rho(x) \leq b w(x)
$$

holds for almost every $x \in X$;

(b)

$$
B \equiv \sup _{0<t<a}\left\|\mu B\left(x_{0}, d\left(x_{0}, \cdot\right)\right)^{-1} \chi_{\left\{d\left(x_{0}, y\right)>t\right\}}\right\|_{L_{v}^{p q}(X)}\left\|\frac{1}{w(\cdot)} \chi_{\left\{d\left(x_{0}, y\right) \leq t\right\}}(\cdot)\right\|_{L_{w}^{p^{\prime} s^{\prime}}(X)}<\infty .
$$

Then there exists a positive constant $c$ such that

$$
\|K f(\cdot)\|_{L_{v}^{p q}(X)} \leq c\|f(\cdot)\|_{L_{w}^{p s}(X)}
$$

for all $f, f \in L_{w}^{p s}(X)$.

Proof. First, let us assume that $\sigma$ is of the kind

$$
\sigma(t)=\sigma(0+)+\int_{0}^{t} \varphi(\tau) d \tau, \quad \varphi \geq 0
$$

Then we have

$$
\begin{gathered}
\|K f(\cdot)\|_{L_{v}^{p q}(X)} \leq c_{1}\left(q \int_{0}^{\infty} \lambda^{q-1}\left(\int_{\{x \in X:|K f(x)|>\lambda\}} \rho(x) \sigma(0+) d \mu\right)^{q / p} d \lambda\right)^{1 / q} \\
+c_{1}\left(q \int_{0}^{\infty} \lambda^{q-1}\left(\int_{\{x \in X:|K f(x)|>\lambda\}} \rho(x)\left(\int_{0}^{d\left(x_{0}, x\right)} \varphi(t) d t\right) d \mu\right)^{q / p} d \lambda\right)^{1 / q} \equiv I_{1}+I_{2} .
\end{gathered}
$$

In the case, where $\sigma(0+)=0$, we have $I_{1}=0$; otherwise, by Theorem B and Lemma A (part (ii)), we have

$$
\begin{aligned}
I_{1} & =c_{1} \sigma(0+)^{1 / p}\|K f(\cdot)\|_{L_{\rho}^{p q}(X)} \leq c_{2} \sigma^{1 / p}(0+)\|f(\cdot)\|_{L_{\rho}^{p q}(X)} \\
& \leq c_{2} \sigma^{1 / p}(0+)\|f(\cdot)\|_{L_{\rho}^{p s}(X)} \leq c_{2}\|f(\cdot)\|_{L_{w}^{p s}(X)} .
\end{aligned}
$$


Now, let us estimate $I_{2}$. Set

$$
f_{1 t}(x)=f(x) \chi_{\left\{d\left(x_{0}, x\right)>\frac{t}{2 a_{1}}\right\}}, \quad f_{2 t}(x)=f(x)-f_{1 t}(x) .
$$

We have

$$
\begin{gathered}
I_{2}=c_{1}\left(q \int_{0}^{\infty} \lambda^{q-1}\left(\int_{0}^{a} \varphi(t)\left(\int_{\left\{x: d\left(x_{0}, x\right)>t,|K f(x)|>\lambda\right\}} \rho(x) d \mu\right) d t\right)^{q / p} d \lambda\right)^{1 / q} \\
\leq c_{3}\left(q \int_{0}^{\infty} \lambda^{q-1}\left(\int_{0}^{a} \varphi(t)\left(\int_{\left\{x: d\left(x_{0}, x\right)>t\right\}} \chi_{\left\{x:\left|K f_{1 t}(x)\right|>\frac{\lambda}{2}\right\}} \rho(x) d \mu\right) d t\right)^{q / p} d \lambda\right)^{1 / q} \\
+c_{3}\left(q \int_{0}^{\infty} \lambda^{q-1}\left(\int_{0}^{a} \varphi(t)\left(\int_{\left\{x: d\left(x_{0}, x\right)>t\right\}} \chi_{\left\{x:\left|K f_{2 t}(x)\right|>\frac{\lambda}{2}\right\}} \rho(x) d \mu\right) d t\right)^{q / p} d \lambda\right)^{1 / q} \equiv I_{21}+I_{22} .
\end{gathered}
$$

Applying the Minkowki's inequality twice $\left(\frac{q}{p} \geq 1, \frac{p}{s} \geq 1\right)$ and using Theorem B and Lemma A (part (ii)), we obtain

$$
\begin{aligned}
I_{21} & \leq c_{4}\left(\int_{0}^{a} \varphi(t)\left(\int_{0}^{\infty} \lambda^{q-1}\left(\int_{\left\{x \in X:\left|K f_{1 t}(x)\right|>\frac{\lambda}{2}\right\}} \rho(x) d \mu\right)^{q / p} d \lambda\right)^{p / q} d t\right)^{1 / p} \\
& \leq c_{5}\left(\int_{0}^{a} \varphi(t)\left\|f_{1 t}(\cdot)\right\|_{L_{\rho}^{p q}(X)}^{p} d t\right)^{1 / p} \leq c_{5}\left(\int_{0}^{a} \varphi(t)\left\|f_{1 t}(\cdot)\right\|_{L_{\rho}^{p s}(X)}^{p} d t\right)^{1 / p} \\
& \leq c_{5}\left(\int_{0}^{\infty} \lambda^{s-1}\left(\int_{\{x \in X:|f(x)|>\lambda\}} \rho(x)\left(\int_{0}^{2 a_{1} d\left(x_{0}, x\right)} \varphi(t) d t\right) d \mu\right)^{s / p} d \lambda\right)^{1 / s} \\
& \leq c_{5}\|f(\cdot)\|_{L_{w}^{p s}(X) \leq c_{6}\|f(\cdot)\|_{L_{w}^{p s}(X)} .}
\end{aligned}
$$

Next we shall estimate $I_{22}$. Note that if $d\left(x_{0}, x\right)>t$ and $d\left(x_{0}, y\right)<\frac{t}{2 a_{1}}$, then

$$
\begin{aligned}
d\left(x_{0}, x\right) & \leq a_{1}\left(d\left(x_{0}, y\right)+d(y, x)\right) \leq a_{1}\left(d\left(x_{0}, y\right)+a_{0} d(x, y)\right) \\
& \leq a_{1}\left(\frac{t}{2 a_{1}}+a_{0} d(x, y)\right) \leq a_{1}\left(\frac{d\left(x_{0}, x\right)}{2 a_{1}}+a_{0} d(x, y)\right) .
\end{aligned}
$$

Hence

and we also have

$$
\frac{d\left(x_{0}, x\right)}{2 a_{1} a_{0}} \leq d(x, y),
$$

$$
\mu\left(B\left(x, d\left(x_{0}, x\right)\right)\right) \leq b \mu\left(B\left(x, \frac{d\left(x_{0}, x\right)}{2 a_{1} a_{0}}\right)\right) \leq b \mu(B(x, d(x, y))) .
$$

Moreover, it is easy to show that

$$
\mu B\left(x_{0}, d\left(x_{0}, x\right)\right) \leq b_{1} \mu B\left(x, d\left(x_{0}, x\right)\right) .
$$

Hence

$$
\mu B\left(x_{0}, d\left(x_{0}, x\right)\right) \leq b_{2} \mu B(x, d(x, y)) .
$$


Taking into account this inequality and using Proposition $\mathrm{C}$ we see that

$$
I_{22} \leq c_{7}\left\|\frac{1}{\mu B\left(x_{0}, d\left(x_{0}, \cdot\right)\right)} \int_{\left\{d\left(x_{0}, y\right)<d\left(x_{0}, \cdot\right)\right\}}|f(y)| d y\right\|_{L_{v}^{p q}(X)} \leq c_{9}\|f(\cdot)\|_{L_{w}^{p s}(X)} .
$$

Now, let $\sigma$ be a positive continuous, but not absolutely continuous, increasing function on $\left(0,4 a_{1} a\right)$; then there exists a sequence of absolutely continuous functions $\sigma_{n}$ such that $\sigma_{n}(t) \leq \sigma(t)$ and $\lim _{n \rightarrow \infty} \sigma_{n}(t)=\sigma(t)$ for arbitrary $t \in$ $\left(0,4 a_{1} a\right)$. For these functions, we can take $\sigma_{n}(t)=\sigma(0+)+n \int_{0}^{t}[\sigma(\tau)-\sigma(\tau-$ $\left.\left.\frac{1}{n}\right)\right] d \tau$.

Denote $v_{n}(x)=\rho(x) \sigma_{n}\left(d\left(x_{0}, x\right)\right)$; then $B_{n}<B$, where

$$
B_{n} \equiv \sup _{t>0}\left\|\chi_{\left\{d\left(x_{0}, y\right)>t\right\}}(\cdot) \mu\left(B\left(x_{0}, d\left(x_{0}, \cdot\right)\right)\right)^{-1}\right\|_{L_{v_{n}}^{p q}(X)}\left\|\frac{1}{w(\cdot)} \chi_{\left\{d\left(x_{0}, y\right) \leq t\right\}}(\cdot)\right\|_{L_{w}^{p^{\prime} s^{\prime}}(X)} .
$$

By virtue of what has been proved, if $B<\infty$, then the following inequality holds:

$$
\|K f(\cdot)\|_{L_{v_{n}}^{p q}(X)} \leq c\|f(\cdot)\|_{L_{w}^{p s}(X)},
$$

where the constant $c>0$ does not depend on $n$.

By passing to the limit as $n \rightarrow \infty$, we obtain inequality (2.1).

Using the representation $\sigma(t)=\sigma(+\infty)+\int_{t}^{\infty} \psi(\tau) d \tau$, where $\sigma(+\infty)=\lim _{t \rightarrow \infty} \sigma(t)$ and $\psi \geq 0$ on $(0, \infty)$ and Proposition $\mathrm{D}$, we obtain the following result.

Theorem 2.2. Let $a=\infty, 1<s \leq p \leq q<\infty$, and suppose that $\sigma$ is a positive decreasing continuous function on $(0, \infty)$. Assume that $\rho \in A_{p}(X)$ and $v(X)=\sigma\left(d\left(x_{0}, x\right)\right) \rho(x)$. Suppose the following conditions are satisfied:

(a) there exists a positive constant $b$ such that the inequality

$$
\rho(x) \sigma\left(\frac{d\left(x_{0}, x\right)}{2 a_{1}}\right) \leq b w(x)
$$

is true for almost every $x \in X$;

(b)

$$
B^{\prime}=\sup _{t>0}\left\|\chi_{\left\{d\left(x_{0}, y\right) \leq t\right\}}(\cdot)\right\|_{L_{v}^{p q}(X)}\left\|\frac{\left(\mu B\left(x_{0}, d\left(x_{0}, x\right)\right)\right)^{-1}}{w(\cdot)} \chi_{\left\{d\left(x_{0}, y\right)>t\right\}}(\cdot)\right\|_{L_{w}^{p^{\prime} s^{\prime}}(X)}<\infty .
$$

Then inequality (2.1) holds.

Now let us consider the particular cases of Theorems 2.1 and 2.2.

Theorem 2.3. Let $a=\infty, 1<p \leq q<\infty$, suppose $\sigma_{1}$ and $\sigma_{2}$ are positive, increasing functions on $(0, \infty)$, let $\sigma_{1}$ be a continuous function and suppose that $\rho \in A_{p}(X)$. Put $v(x)=\sigma_{2}\left(d\left(x_{0}, x\right)\right) \rho(x), w(x)=\sigma_{1}\left(d\left(x_{0}, x\right)\right) \rho(x)$. If

$$
\sup _{t>0}\left\|\left(\mu B\left(x_{0}, d\left(x_{0}, \cdot\right)\right)\right)^{-1} \chi_{\left\{d\left(x_{0}, y\right)>t\right\}}(\cdot)\right\|_{L_{v}^{p q}(X)}\left\|\frac{1}{w(\cdot)} \chi_{\left\{d\left(x_{0}, y\right) \leq t\right\}}(\cdot)\right\|_{L_{w}^{p^{\prime}}(X)}<\infty,
$$


then there exists a positive constant $c$ such that

$$
\|K f(\cdot)\|_{L_{v}^{p q}(X)} \leq c\|f(\cdot)\|_{L_{w}^{p}(X)}
$$

for all $f \in L_{w}^{p}(X)$.

Proof. By Theorem 2.1 it is sufficient to show that there exists a positive constant $b$ such that $\sigma_{2}\left(2 a_{1} t\right) \leq b \sigma_{1}(t)$ for all $t \in(0, \infty)$.

From the doubling condition (see (vii) in Definition 1.1) and the condition (1.1) it follows that the measure $\mu$ satisfies the reverse doubling condition at the point $x_{0}$ (see, e.g., [20] and [23], Lemma 20). In other words, there exist constants $\eta_{1}>1$ and $\eta_{2}>1$ such that

$$
\mu\left(B\left(x_{0}, \eta_{1} r\right)\right) \geq \eta_{2} \mu\left(B\left(x_{0}, r\right)\right)
$$

for all $r>0$.

Applying the Hölder's inequality and using Lemma $\mathrm{C}$, the reverse doubling condition and the fact that $\rho^{1-p^{\prime}} \in A_{p^{\prime}}(X)$, we obtain

$$
\begin{gathered}
\frac{\sigma_{2}\left(2 a_{1} t\right)}{\sigma_{1}(t)} \leq\left(\mu\left(B\left(x_{0}, 2 a_{1} \eta_{1} t\right) \backslash B\left(x_{0}, 2 a_{1} t\right)\right)\right)^{-p}\left(\int_{B\left(x_{0}, 2 a_{1} \eta_{1} t\right) \backslash B\left(x_{0}, 2 a_{1} t\right)} \rho(x) d \mu\right) \\
\times\left(\int_{B\left(x_{0}, 2 a_{1} \eta_{1} t\right) \backslash B\left(x_{0}, 2 a_{1} t\right)} \rho^{1-p^{\prime}}(x) d \mu\right)^{p-1} \frac{\sigma_{2}\left(2 a_{1} t\right)}{\sigma_{1}(t)} \\
\leq\left(\left(1-\frac{1}{\eta_{2}}\right) \mu B\left(x_{0}, 2 a_{1} \eta t\right)\right)^{-p}\left(\int_{B\left(x_{0}, 2 a_{1} \eta_{1} t\right) \backslash B\left(x_{0}, 2 a_{1} t\right)} \rho(x) d \mu\right) \\
\times\left(\int_{B\left(x_{0}, t\right)} \rho^{1-p^{\prime}}(x) d \mu\right)^{p-1} \frac{\sigma_{2}\left(2 a_{1} t\right)}{\sigma_{1}(t)} \\
\leq c_{1}\left(\mu B\left(x_{0}, 2 a_{1} \eta t\right)\right)^{-p}\left\|\chi_{\left(B\left(x_{0}, 2 a_{1} \eta_{1} t\right) \backslash B\left(x_{0}, 2 a_{1} t\right)\right)}(\cdot)\right\|_{L_{v}^{p q}(X)}^{p}\left\|\frac{1}{w(\cdot)} \chi_{B\left(x_{0}, t\right)}(\cdot)\right\|_{L_{w}^{p^{\prime}}(X)}^{p} \\
\leq c_{2}\left\|\left(\mu B\left(x_{0}, d\left(x_{0}, \cdot\right)\right)\right)^{-1} \chi_{\left\{d\left(x_{0}, y\right)>t\right\}}(\cdot)\right\|_{L_{v}^{p q}(X)}^{p}\left\|\frac{1}{w(\cdot)} \chi_{B\left(x_{0}, t\right)}(\cdot)\right\|_{L_{w}^{p^{\prime}}(X)}^{p} \leq c .
\end{gathered}
$$

An analogous theorem dealing with singular integrals on homogeneous groups was proved in [16], and for singular integrals on SHT in the Lebesgue space in [5].

Theorem 2.4. Let $a=\infty, 1<s \leq p \leq q<\infty$; suppose $\sigma_{1}, \sigma_{2}, u_{1}$ and $u_{2}$ are weight functions defined on $X$. Let $\rho \in A_{p}(X)$, and suppose $v=\sigma_{2} \rho$, $w=\sigma_{1} \rho$. Assume that the following conditions are fulfilled:

(1) there exists a positive constant $b$ such that for all $t>0$

$$
\sup _{F_{t}} \sigma_{2}^{1 / p}(x) \sup _{F_{t}} u_{2}(x) \leq b \inf _{F_{t}} \sigma_{1}^{1 / p}(x) \inf _{F_{t}} u_{1}(x)
$$

holds, where $F_{t}=\left\{x \in X: \frac{t}{a_{1}} \leq d\left(x_{0}, x\right)<8 a_{1} t\right\}$; 
$(2)$

$$
\begin{gathered}
\sup _{t>0}\left\|u_{2}(\cdot)\left(\mu\left(B\left(x_{0}, d\left(x_{0}, \cdot\right)\right)\right)\right)^{-1} \chi_{\left\{d\left(x_{0}, t\right)>t\right\}}(\cdot)\right\|_{L_{v}^{p q}(X)} \\
\times\left\|\frac{1}{u_{1}(\cdot) w(\cdot)} \chi_{\left\{d\left(x_{0}, y\right) \leq t\right\}}(\cdot)\right\|_{L_{w}^{p^{\prime} s^{\prime}}(X)}<\infty ;
\end{gathered}
$$

$$
\sup _{t>0}\left\|u_{2}(\cdot) \chi_{\left\{d\left(x_{0}, t\right) \leq t\right\}}(\cdot)\right\|_{L_{v}^{p q}(X)}\left\|\frac{\left(\mu B\left(x_{0}, d\left(x_{0}, \cdot\right)\right)\right)^{-1}}{u_{1}(\cdot) w(\cdot)} \chi_{\left\{d\left(x_{0}, y\right)>t\right\}}(\cdot)\right\|_{L_{w}^{p^{\prime} s^{\prime}}(X)}<\infty .
$$

Then

$$
\left\|u_{2}(\cdot) K f(\cdot)\right\|_{L_{v}^{p q}(X)} \leq c\left\|u_{1}(\cdot) f(\cdot)\right\|_{L_{w}^{p s}(X)},
$$

where the positive constant $c$ does not depend on $f$.

Proof. Let

$$
\begin{gathered}
E_{k} \equiv B\left(x_{0}, 2^{k+1}\right) \backslash B\left(x_{0}, 2^{k}\right), \quad G_{k, 1} \equiv B\left(x_{0}, 2^{k-1} / a_{1}\right), \\
G_{k, 2} \equiv B\left(x_{0}, a_{1} 2^{k+2}\right) \backslash B\left(x_{0}, 2^{k-1} / a_{1}\right), \quad G_{k, 3} \equiv X \backslash B\left(x_{0}, a_{1} 2^{k+2}\right) .
\end{gathered}
$$

We obtain

$$
\begin{gathered}
\left\|u_{2}(\cdot) K f(\cdot)\right\|_{L_{v}^{p q}(X)}^{p} \leq c_{1}\left\|\sum_{k \in Z} u_{2}(\cdot) K\left(f \chi_{G_{k, 1}}\right)(\cdot) \chi_{E_{k}}(\cdot)\right\|_{L_{v}^{p q}(X)}^{p} \\
+c_{1}\left\|\sum_{k \in Z} u_{2}(\cdot) K\left(f \chi_{G_{k, 2}}\right)(\cdot) \chi_{E_{k}}(\cdot)\right\|_{L_{v}^{p q}(X)}^{p} \\
+c_{1}\left\|\sum_{k \in Z} u_{2}(\cdot) K\left(f \chi_{G_{k, 3}}\right)(\cdot) \chi_{E_{k}}(\cdot)\right\|_{L_{v}^{p q}(X)}^{p} \equiv c_{1}\left(S_{1}^{p}+S_{2}^{p}+S_{3}^{p}\right) .
\end{gathered}
$$

Now we estimate $S_{1}$. Note that

$$
d\left(x_{0}, y\right)<\frac{2^{k-1}}{a_{1}} \leq \frac{d\left(x_{0}, x\right)}{2 a_{1}}
$$

when $x \in E_{k}$, and $y \in G_{k, 1}$. From the latter inequality we have

$$
\mu B\left(x, d\left(x_{0}, x\right)\right) \leq b_{1} \mu B(x, d(x, y)) .
$$

Indeed,

$$
d\left(x_{0}, x\right) \leq a_{1}\left(d\left(x_{0}, y\right)+d(y, x)\right) \leq a_{1}\left(\frac{d\left(x_{0}, x\right)}{2 a_{1}}+a_{0} d(x, y)\right) .
$$

Hence

$$
\frac{1}{2 a_{1} a_{0}} d\left(x_{0}, x\right) \leq d(x, y)
$$

Correspondingly,

$$
\mu B\left(x, d\left(x_{0}, x\right)\right) \leq b_{2} \mu B\left(x, \frac{d\left(x_{0}, x\right)}{2 a_{1} a_{0}}\right) \leq b_{2} \mu B(x, d(x, y)) .
$$


It is easy to see that

$$
\mu B\left(x_{0}, d\left(x_{0}, x\right)\right) \leq b_{3} \mu B\left(x, d\left(x_{0}, x\right)\right)
$$

and, finally, we obtain

$$
\mu B\left(x_{0}, d\left(x_{0}, x\right)\right) \leq b_{4} \mu B(x, d(x, y)) .
$$

By considering the latter inequality we have

$$
\begin{aligned}
\left|K\left(f \chi_{G_{k 1}}\right)(x)\right| & \leq b_{5} \int_{X} \frac{|f(y)| \chi_{G_{k 1}}(y)}{\mu B(x, d(x, y))} d \mu \\
& \leq \frac{b_{6}}{\mu B\left(x_{0}, d\left(x_{0}, x\right)\right)} \int_{B\left(x_{0}, d\left(x_{0}, x\right)\right)}|f(y)| d \mu,
\end{aligned}
$$

when $x \in E_{k}$, and by Proposition $\mathrm{C}$ we obtain

$$
\begin{aligned}
S_{1}^{p} & \leq c_{2}\left\|u_{2}(\cdot)\left(\mu B\left(x_{0}, d\left(x_{0}, \cdot\right)\right)\right)^{-1} \int_{B\left(x_{0}, d\left(x_{0}, x\right)\right)}|f(y)| d \mu\right\|_{L_{v}^{p q}(X)}^{p} \\
& \leq c_{3}\left\|u_{1}(\cdot) f(\cdot)\right\|_{L_{w}^{p s}(X)}^{p} .
\end{aligned}
$$

Now we shall estimate $S_{3}^{p}$. It is easy to check that if $x \in E_{k}$ and $y \in G_{k, 3}$, then $d\left(x_{0}, y\right) \leq d(x, y)$ and

$$
\mu B\left(x_{0}, d\left(x_{0}, y\right)\right) \leq b_{7} \mu B(x, d(x, y)) .
$$

By virtue of Proposition D we obtain

$$
S_{3}^{p} \leq c_{4}\left\|u_{2}(\cdot) \int_{\left\{d\left(x_{0}, y\right)>d\left(x_{0}, x\right)\right\}} \frac{|f(y)|}{\mu B\left(x_{0}, d\left(x_{0}, y\right)\right)} d \mu\right\|_{L_{v}^{p q}(X)}^{p} \leq c_{5}\left\|u_{1}(\cdot) f(\cdot)\right\|_{L_{w}^{p q}(X)}^{p} .
$$

Now let us estimate $S_{2}^{p}$. By Lemma B (part (ii)),

$$
S_{2}^{p} \leq \sum_{k \in u}\left\|u_{2}(\cdot) K\left(f \chi_{G_{k, 2}}\right)(\cdot) \chi_{E_{k}}(\cdot)\right\|_{L_{v}^{p q}(X)}^{p} \equiv \sum_{k \in u} S_{k, 2}^{p} .
$$

We shall use the following notation:

$$
u_{2, k} \equiv \sup _{x \in E_{k}} u_{2}(x), \quad \sigma_{2, k} \equiv \sup _{x \in E_{k}} \sigma_{2}(x), \quad u_{1, k} \equiv \inf _{x \in G_{k, 2}} u_{1}(x), \quad \sigma_{1, k} \equiv \inf _{x \in G_{k, 2}} \sigma_{1}(x) .
$$

By Theorem $\mathrm{C}$ and Lemma A we have

$$
\begin{aligned}
S_{k, 2} & \leq u_{2, k} \sigma_{2, k}^{1 / p}\left\|K\left(f \chi_{G_{k, 2}}\right)(\cdot)\right\|_{L_{\rho}^{p q}(X)} \leq c_{6} u_{2, k} \sigma_{2, k}^{1 / p}\left\|f(\cdot) \chi_{G_{k, 2}}(\cdot)\right\|_{L_{\rho}^{p q}(X)} \\
& \leq c_{6} u_{2, k} \sigma_{2, k}^{1 / p}\left\|f(\cdot) \chi_{G_{k, 2}}(\cdot)\right\|_{L_{\rho}^{p s}(X)} \leq c_{7} u_{1, k} \sigma_{1, k}^{1 / p}\left\|f(\cdot) \chi_{G_{k, 2}}(\cdot)\right\|_{L_{\rho}^{p s}(X)}^{p} \\
& \leq c_{8}\left\|u_{1}(\cdot) f(\cdot) \chi_{G_{k, 2}}(\cdot)\right\|_{L_{w}^{p s}(X)} .
\end{aligned}
$$

By Lemma B (part (i)) we finally obtain

$$
S_{2}^{p} \leq c_{9}\left\|u_{1}(\cdot) f(\cdot)\right\|_{L_{w}^{p s}(X)} .
$$


Remark 2.1. It is easy to check that Theorem 2.4 is still valid if we replace condition (1) by the condition

$\left(1^{\prime}\right)$

$$
\sup _{E_{x}} \sigma_{2}(y) \sup _{E_{x}} u_{2}^{p}(y) \leq \bar{b} \sigma_{1}(x) u_{1}^{p}(x),
$$

where $\bar{b}>0$ does not depend on $x \in X$ and where

$$
E_{x}=\left\{y: \frac{d\left(x_{0}, x\right)}{4 a_{1}} \leq d\left(x_{0}, y\right)<4 a_{1} d\left(x_{0}, x\right)\right\} .
$$

Indeed, we have

$$
\begin{aligned}
S_{k, 2} & \leq \sigma_{2, k}^{1 / p} u_{2, k}\left\|T\left(f \chi_{G_{k, 2}}\right)(\cdot)\right\|_{L_{\rho}^{p q}(X)} \leq \sigma_{2, k}^{1 / p} u_{2, k}\left\|T\left(f \chi_{G_{k, 2}}\right)(\cdot)\right\|_{L_{\rho}^{p}(X)} \\
& \leq b_{1} \sigma_{2, k}^{1 / p} u_{2, k}\left\|f(\cdot) \chi_{G_{k, 2}}(\cdot)\right\|_{L_{\rho}^{p}(X)} \\
& \left.=b_{1} \int_{G_{k, 2}}\left(\sup _{2^{k} \leq d\left(x_{0}, y\right)<2^{k+1}} \sigma_{2}(y)\right)\left(\sup _{2^{k} \leq d\left(x_{0}, y\right)<2^{k+1}} u_{2}^{p}(y)\right)|f(x)|^{p} \rho(x) d \mu\right)^{1 / p} \\
& \leq b_{1}\left(\int_{G_{k, 2}}\left(\sup _{E_{x}} \sigma_{2}(y)\right)\left(\sup _{E_{x}} u_{2}^{p}(y)\right)|f(x)|^{p} \rho(x) d \mu\right)^{1 / p} \\
& \leq b_{2}\left(\int_{G_{k, 2}} \sigma_{1}(x) u_{1}^{p}(x) \rho(x)|f(x)|^{p} d \mu\right)^{1 / p} \\
& =b_{2}\left\|f(\cdot) u_{1}(\cdot) \chi_{G_{k, 2}}(\cdot)\right\|_{L_{w}^{p}(X)} \leq b_{2}\left\|f(\cdot) u_{1}(\cdot) \chi_{G_{k, 2}}(\cdot)\right\|_{L_{w}^{s p}(X)} .
\end{aligned}
$$

In [19] it is proved that conditions (2) and (3) of Theorem 2.4 are also necessary for equality (2.2) to be fulfilled when $K$ is the Hilbert transform.

Theorem 2.5. Let $\mu(X)=\infty, 1<s \leq p \leq q$ and suppose $\varphi_{1}, \varphi_{2}$, and $v$ are positive increasing functions on $(0, \infty)$.

If the condition

$$
\begin{aligned}
\sup _{t>0} B(t) & \equiv \sup _{t>0}\left\|\varphi_{2}\left(d\left(x_{0}, \cdot\right)\right)\left(\mu B\left(x_{0}, d\left(x_{0}, x\right)\right)\right)^{-1} \chi_{\left\{d\left(x_{0}, y\right)>t\right\}}(\cdot)\right\|_{L_{v\left(d\left(x_{0}, \cdot\right)\right)}^{p q}(X)} \\
& \times\left\|\frac{1}{\varphi_{1}\left(d\left(x_{0}, \cdot\right)\right)} \chi_{\left\{d\left(x_{0}, y\right) \leq t\right\}}(\cdot)\right\|_{L^{p^{\prime} s^{\prime}}(X)}<\infty
\end{aligned}
$$

is fulfilled, then the following weighted inequality holds:

$$
\left\|K f(\cdot) \varphi_{2}\left(d\left(x_{0}, \cdot\right)\right)\right\|_{L_{v\left(d\left(x_{0}, \cdot\right)\right)}^{p q}(X)} \leq c\left\|f(\cdot) \varphi_{1}\left(d\left(x_{0}, \cdot\right)\right)\right\|_{L^{p s}(X)} .
$$

Proof. First, let us prove the inequality

$$
\varphi_{2}\left(8 a_{1} t\right) v^{1 / p}\left(8 a_{1} t\right) \leq b_{1} \varphi_{1}\left(\frac{t}{a_{1}}\right)
$$


where the positive constant $b_{1}$ does not depend on $t>0$. Indeed, by Lemma A (part (i)) and by the reverse doubling condition for $\mu$ we have

$$
\begin{aligned}
c \geq B(t) & \geq \| \varphi_{2}\left(d\left(x_{0}, \cdot\right)\right)\left(\mu B\left(x_{0}, d\left(x_{0}, \cdot\right)\right)^{-1} \chi_{\left\{t<d\left(x_{0}, y\right)<\eta_{1} t\right\}}(\cdot) \|_{L_{v\left(d\left(x_{0}, \cdot\right)\right)}^{p s}(X)}\right. \\
& \times\left\|\frac{1}{\varphi_{1}\left(d\left(x_{0}, \cdot\right)\right)} \chi_{\left\{d\left(x_{0}, y\right) \leq \frac{t}{\left.8 a_{1}^{2}\right\}}(\cdot)\right.}\right\|_{L^{p^{\prime} s^{\prime}(X)}} \\
& \geq c_{1}\left(\mu B\left(x_{0}, \eta_{1} t\right)\right)^{-1} \varphi_{2}(t)\left(\int_{\left\{t<d\left(x_{0}, y\right)<\eta_{1} t\right\}} v\left(d\left(x_{0}, y\right)\right) d \mu\right)^{1 / p} \\
& \times \varphi_{1}^{-1}\left(\frac{t}{8 a_{1}^{2}}\right)\left(\mu B\left(x_{0}, \frac{t}{8 a_{1}^{2}}\right)\right)^{1 / p} \\
& \geq c_{2} \varphi_{2}(t) v^{1 / p}(t) \mu B\left(x_{0}, t\right)^{-1} \mu B\left(x_{0}, t\right)^{1 / p^{\prime}} \varphi_{1}^{-1}\left(\frac{t}{8 a_{1}^{2}}\right) \mu B\left(x_{0}, t\right)^{1 / p^{\prime}} \\
& =c_{2} \frac{\varphi_{2}(t) v^{1 / p}(t)}{\varphi_{1}\left(\frac{t}{8 a_{1}^{2}}\right)} .
\end{aligned}
$$

Now, we are to show that the following condition is fulfilled:

$$
\begin{aligned}
\sup _{t>0} B_{1}(t) & \equiv \sup _{t>0}\left\|\varphi_{2}\left(d\left(x_{0}, \cdot\right)\right) \chi_{\left\{d\left(x_{0}, y\right) \leq t\right\}}(\cdot)\right\|_{L_{v}^{p q}(X)} \\
& \times\left\|\frac{\mu B\left(x_{0}, d\left(x_{0}, \cdot\right)\right)^{-1}}{\varphi_{1}\left(d\left(x_{0}, \cdot\right)\right)} \chi_{\left\{d\left(x_{0}, y\right)>t\right\}}(\cdot)\right\|_{L^{p^{\prime} s^{\prime}}(X)}<\infty .
\end{aligned}
$$

Indeed, by the monotonic property of the functions $\varphi_{1}, \varphi_{2}$, and $v$ and by Lemma A (part (i)), we obtain:

$$
\begin{aligned}
B_{1}(t) & \leq c_{3} \varphi_{2}(t)\left(\mu B\left(x_{0}, t\right)\right)^{1 / p} v^{1 / p}(t) \varphi_{1}^{-1}\left(\frac{t}{8 a_{1}^{2}}\right) \\
& \times \|\left(\mu B\left(x_{0}, d\left(x_{0}, \cdot\right)\right)^{-1} \chi_{\left\{d\left(x_{0}, y\right)>t\right\}}(\cdot) \|_{L^{p^{\prime} s^{\prime}}(X)} .\right.
\end{aligned}
$$

On the other hand, we have

$$
\begin{gathered}
\|\left(\mu B\left(x_{0}, d\left(x_{0}, \cdot\right)\right)^{-1} \chi_{\left\{d\left(x_{0}, y\right)>t\right\}}(\cdot) \|_{L^{p^{\prime} s^{\prime}}(X)}\right. \\
=\left(s^{\prime} \int_{0}^{\infty} \lambda^{s^{\prime}-1}\left(\mu\left\{x:\left(\mu B\left(x_{0}, d\left(x_{0}, x\right)\right)^{-1}>\lambda\right\} \cap\left\{d\left(x_{0}, x\right) \geq t\right\}\right)\right)^{s^{\prime} / p^{\prime}} d \lambda\right)^{1 / s^{\prime}} \\
=\left(s^{\prime} \int_{0}^{\left(\mu B\left(x_{0}, t\right)\right)^{-1}} \lambda^{s^{\prime}-1}\left(\mu\left\{x: \mu B\left(x_{0}, d\left(x_{0}, x\right)\right)<\lambda^{-1}\right\}\right)^{s^{\prime} / p^{\prime}} d \lambda\right)^{1 / s^{\prime}} \\
\leq c_{4}\left(\int_{0}^{\left(\mu B\left(x_{0}, t\right)\right)^{-1}} \lambda^{s^{\prime}-1} \lambda^{-s^{\prime} / p^{\prime}} d \lambda\right)^{1 / s^{\prime}}=c_{5}\left(\mu B\left(x_{0}, t\right)\right)^{-1 / p} .
\end{gathered}
$$


Here we have used the inequality

$$
\mu\left\{x: \mu B\left(x_{0}, d\left(x_{0}, x\right)\right)<\lambda^{-1}\right\} \leq b \lambda^{-1},
$$

where the positive constant $b$ is from the doubling condition for $\mu$. Thus we obtain

$$
B_{1}(t) \leq c_{6} \varphi_{2}(t) v^{1 / p}(t) \varphi_{1}^{-1}\left(\frac{t}{8 a_{1}^{2}}\right) \leq c_{7}
$$

for arbitrary $t>0$.

By Theorem 2.4 we conclude that inequality (2.3) holds.

Remark 2.2. All results of this section remain valid if we omit the continuity of $\sigma$, but require that $\mu B\left(x_{0}, r\right)$ be continuous with respect to $r$, where $x_{0}$ is the same fixed point as above. This follows from the fact that in this case the sequence $\sigma_{n}\left(d\left(x_{0}, x\right)\right)$, where $\sigma_{n}$ are absolutely continuous functions (see the proof of Theorem 2.1.), converges to $\sigma\left(d\left(x_{0}, x\right)\right)$ a.e. on $X$. On the other hand, the continuity of $\mu B\left(x_{0}, r\right)$ with respect to $r$ is equivalent to the condition $\mu\left\{x: d\left(x_{0}, x\right)=r\right\}=0$ for all $r>0$.

\section{Singular Integrals on Spaces of Nonhomogeneous Type}

In this section we present weighted inequalities for Calderón-Zygmund singular integrals defined on spaces of nonhomogeneous type.

Let $(X, d, \mu)$ be a spaces of nonhomogeneous type with metric $d$ and measure $\mu$ (i.e., $a_{0}=1, a_{1}=1$, we have equality instead of the inequality in (iii) and (vii) need not be valid in Definition 1.1) satisfying the condition

$$
\mu \bar{B}(x, r) \leq r^{\alpha}, \quad x \in X, \quad r>0,
$$

for some $\alpha>0$, where $\bar{B}(x, r) \equiv\{y: d(x, y) \leq r\}$.

Let the kernel $k$ satisfy the following conditions:

$$
|k(x, y)| \leq c_{1} d(x, y)^{-\alpha}
$$

for all $x, y \in X, x \neq y$;

(2) there exist $c_{2}>0$ and $\varepsilon \in(0,1]$ such that

$$
\max \left\{\left|k(x, y)-k\left(x_{1}, y\right)\right|,\left|k(y, x)-k\left(y, x_{1}\right)\right|\right\} \leq c_{2} \frac{d\left(x, x_{1}\right)^{\varepsilon}}{d(x, y)^{\alpha+\varepsilon}}
$$

whenever $d\left(x, x_{1}\right) \leq 2^{-1} d(x, y), x \neq y$. Assume also that the integral operator

$$
T f(x)=\lim _{\varepsilon \rightarrow 0} \int_{X \backslash B\left(x_{0}, \varepsilon\right)} f(y) k(x, y) d \mu
$$

is bounded in $L^{2}(X)$.

The following theorem is valid.

Theorem $\mathbf{C}$ ([18]). The operator $T$ is bounded in $L^{p}(X)$ for $1<p<\infty$ and is of weak type $(1,1)$. 
Recall that $a \equiv \sup \left\{d\left(x_{0}, x\right): x \in X\right\}$, where $x_{0}$ is a given point of $X$ and $B\left(x_{0}, R\right) \backslash B\left(x_{0}, r\right) \neq \varnothing$ whenever $0<r<R<a$.

We remark that the condition $\mu\{x\}=0$ is automatically satisfied for all $x \in X$.

Lemma 3.1. Let $1<p<\infty$, and let $w$ be a weight function on $X$. Assume that the following two conditions are satisfied:

(i) there exists a positive increasing function $v$ on $(0,4 a)$ such that for almost all $x \in X$ the inequality

$$
v\left(2 d\left(x_{0}, x\right)\right) \leq b_{1} w(x)
$$

holds, where the positive constant $b_{1}$ does not depend on $x$;

(ii)

$$
I(t) \equiv \int_{B\left(x_{0}, t\right)} w^{1-p^{\prime}}(x) d \mu<\infty
$$

for all $t>0$. Then $T \varphi(x)$ exists $\mu$-a.e. for any $\varphi \in L_{w}^{p}(X)$.

Proof. Let $0<\alpha<a$ and let us denote

$$
S_{\beta}=\left\{x \in X: d\left(x_{0}, x\right) \geq \beta / 2\right\} .
$$

Suppose $\varphi \in L_{w}^{p}(X)$ and represent $\varphi$ as follows:

$$
\varphi(x)=\varphi_{1}(x)+\varphi_{2}(x)
$$

where $\varphi_{1}=\varphi \chi_{S_{\beta}}$ and $\varphi_{2}=\varphi-\varphi_{1}$. Due to condition (i) we see that

$$
\begin{gathered}
\int_{X}\left|\varphi_{1}(x)\right|^{p} d \mu=\frac{v\left(\frac{\beta}{2}\right)}{v\left(\frac{\beta}{2}\right)} \int_{S_{\beta}}|\varphi(x)|^{p} d \mu \\
\leq \frac{1}{v\left(\frac{\beta}{2}\right)} \int_{S_{\beta}}|\varphi(x)|^{p} v\left(2 d\left(x_{0}, x\right)\right) d \mu \leq \frac{c_{1}}{v\left(\frac{\beta}{2}\right)} \int_{S_{\beta}}|\varphi(x)|^{p} w(x) d \mu<\infty
\end{gathered}
$$

for arbitrary $\beta, 0<\beta<a$. Consequently $T \varphi_{1} \in L^{p}(X)$ and, according to Theorem C, $T \varphi_{1}(x)$ exists $\mu$-a.e. on $X$.

Now let $x$ be such that $d\left(x_{0}, x\right)>\beta$. If $y \in X$ and $d\left(x_{0}, y\right)<\frac{\beta}{2}$, then

$$
d\left(x_{0}, x\right) \leq d\left(x_{0}, y\right)+d(x, y) \leq \frac{d\left(x_{0}, x\right)}{2}+d(x, y)
$$


Hence $\beta / 2<\frac{d\left(x_{0}, x\right)}{2} \leq d(x, y)$ and we obtain

$$
\begin{gathered}
\left|T \varphi_{2}(x)\right|=\left|\int_{X} \varphi_{2}(y) k(x, y) d \mu\right| \leq c_{2} \int_{B\left(x_{0}, \frac{\beta}{2}\right)} \frac{|\varphi(y)|}{d(x, y)^{\alpha}} d \mu \\
\leq c_{3} \int_{B\left(x_{0}, \frac{\beta}{2}\right)} \frac{|\varphi(y)|}{d(x, y)^{\alpha}} d \mu \leq c_{4} \beta^{-\alpha} \int_{B\left(x_{0}, \frac{\beta}{2}\right)}|\varphi(y)| d \mu \\
\leq c_{4} \beta^{-\alpha}\left(\int_{B\left(x_{0}, \frac{\beta}{2}\right)}|\varphi(y)|^{p} w(y) d \mu\right)^{1 / p}\left(\int_{B\left(x_{0}, \frac{\beta}{2}\right)} w^{1-p^{\prime}}(y) d \mu\right)^{1 / p^{\prime}}<\infty .
\end{gathered}
$$

Thus $T \varphi(x)$ is absolutely convergent for arbitrary $x$ such that $d\left(x_{0}, x\right)>\beta$. As we can take $\beta$ arbitrarily small and $\mu\left\{x_{0}\right\}=0$, we conclude that $T \varphi(x)$ exists $\mu$-a.e. on $X$.

Theorem 3.1. Let $1<p<\infty$. Assume that $v$ is a positive increasing continuous function on $(0,4 a)$. Suppose that $w$ is a weight on $X$. Let the following two conditions hold:

(i) there exists a constant $b_{1}>0$ such that the inequality

$$
v\left(2 d\left(x_{0}, x\right)\right) \leq b_{1} w(x)
$$

is fulfilled for $\mu$-almost all $x \in X$;

(ii)

$$
\sup _{0<t<a}\left(\int_{X \backslash B\left(x_{0}, t\right)} \frac{v\left(d\left(x_{0}, x\right)\right)}{d\left(x_{0}, x\right)^{\alpha p}} d \mu\right)^{1 / p}\left(\int_{B\left(x_{0}, t\right)} w^{1-p^{\prime}}(x) d \mu\right)^{1 / p^{\prime}}<\infty .
$$

Then $T$ is bounded from $L_{w}^{p}(X)$ to $L_{v\left(d\left(x_{0}, \cdot\right)\right)}^{p}(X)$.

Proof. Without loss of generality we can suppose that $v$ has the form

$$
v(t)=v(0+)+\int_{0}^{t} \phi(\tau) d \tau, \quad \phi \geq 0
$$

We have

$$
\begin{gathered}
\int_{X}|T f(x)|^{p} v\left(d\left(x_{0}, x\right)\right) d \mu=v(0+) \int_{X}|T f(x)|^{p} d \mu \\
+\int_{X}|T f(x)|^{p}\left(\int_{0}^{d\left(x_{0}, x\right)} \phi(t) d t\right) d \mu \equiv I_{1}+I_{2} .
\end{gathered}
$$

If $v(0+)=0$, then $I_{1}=0$. If $v(0+) \neq 0$, by Theorem $\mathrm{C}$ we obtain

$$
I_{1} \leq c_{1} v(0+) \int_{X}|f(x)|^{p} d \mu \leq c_{1} \int_{X}|f(x)|^{p} v\left(d\left(x_{0}, x\right)\right) d \mu \leq c_{2} \int_{X}|f(x)|^{p} w(x) d x .
$$


Changing the order of integration in $I_{2}$, we have

$$
\begin{gathered}
I_{2}=\int_{0}^{a} \phi(t)\left(\int_{\left\{x: d\left(x_{0}, x\right)>t\right\}}|T f(x)|^{p} d \mu\right) d t \\
\leq c_{3} \int_{0}^{a} \phi(t)\left(\int_{\left\{x: d\left(x_{0}, x\right)>t\right\}}\left|\int_{\left\{y: d\left(x_{0}, y\right)>\frac{t}{2}\right\}} f(y) k(x, y) d \mu\right|^{p} d \mu\right) d t \\
+c_{3} \int_{0}^{a} \phi(t)\left(\int_{\left\{x: d\left(x_{0}, x\right)>t\right\}}\left|\int_{\left\{y: d\left(x_{0}, y\right) \leq \frac{t}{2}\right\}} f(y) k(x, y) d \mu\right|^{p} d \mu\right) d t \equiv I_{21}+I_{22} .
\end{gathered}
$$

Using again the boundedness of $T$ in $L^{p}(X)$ we obtain

$$
\begin{gathered}
I_{21} \leq c_{4} \int_{0}^{a} \phi(t)\left(\int_{\left\{y: d\left(x_{0}, y\right)>\frac{t}{2}\right\}}|f(y)|^{p} d \mu\right) d t=c_{4} \int_{X}|f(y)|^{p}\left(\int_{0}^{2 d\left(x_{0}, y\right)} \phi(t) d t\right) d \mu \\
\leq c_{4} \int_{X}|f(y)|^{p} v\left(2 a_{1} d\left(x_{0}, y\right)\right) d \mu \leq c_{5} \int_{X}|f(y)|^{p} w(y) d \mu .
\end{gathered}
$$

Now let us estimate $I_{22}$. When $d\left(x_{0}, x\right)>t$ and $d\left(x_{0}, y\right) \leq \frac{t}{2}$ we have

$$
\begin{aligned}
d\left(x_{0}, x\right) & \leq d\left(x_{0}, y\right)+d(y, x)=d\left(x_{0}, y\right)+d(x, y) \\
& \leq \frac{t}{2}+d(x, y) \leq \frac{d\left(x_{0}, x\right)}{2}+d(x, y) .
\end{aligned}
$$

Hence

$$
\frac{d\left(x_{0}, x\right)}{2} \leq d(x, y)
$$

Consequently,

$$
\begin{aligned}
I_{22} & \leq c_{6} \int_{0}^{a} \phi(t)\left(\int_{\left\{x: d\left(x_{0}, y\right)>t\right\}}\left(\int_{\left\{y: d\left(x_{0}, y\right) \leq \frac{t}{2}\right\}} \frac{|f(y)|}{d(x, y)^{\alpha}} d \mu(y)\right)^{p} d \mu(x)\right) d t \\
& \leq c_{7} \int_{0}^{a} \phi(t)\left(\int_{\left\{x: d\left(x_{0}, x\right)>t\right\}} \frac{1}{d\left(x_{0}, x\right)^{\alpha p}} d \mu\right)\left(\int_{B\left(x_{0}, t\right)}|f(y)| d \mu\right)^{p} d t .
\end{aligned}
$$

It is easy to see that for any $s, 0<s<a$, we have

$$
\begin{gathered}
\int_{s}^{a} \phi(t)\left(\int_{\left\{x: d\left(x_{0}, x\right)>t\right\}} \frac{1}{d\left(x_{0}, x\right)^{\alpha p}} d \mu\right) d t \\
\leq \int_{\left\{x: d\left(x_{0}, x\right) \geq s\right\}} \frac{1}{d\left(x_{0}, x\right)^{\alpha p}}\left(\int_{s}^{d\left(x_{0}, x\right)} \phi(t) d t\right) d \mu \leq \int_{\left\{x: d\left(x_{0}, x\right) \geq s\right\}} \frac{v\left(d\left(x_{0}, x\right)\right)}{d\left(x_{0}, x\right)^{\alpha p}} d \mu .
\end{gathered}
$$


Finally, using Proposition A, we obtain

$$
I_{22} \leq c_{8} \int_{X}|f(x)|^{p} w(x) d \mu .
$$

Lemma 3.2. Let $a=\infty, 1<p<\infty$, and let $w$ be a weight function on $X$. Suppose the following conditions are fulfilled:

(i) there exists a positive decreasing function $v$ on $(0, \infty)$ such that

$$
v\left(\frac{d\left(x_{0}, x\right)}{2}\right) \leq c w(x) \quad \text { a.e. }
$$

(ii) for all $t>0$

$$
\int_{X \backslash B\left(x_{0}, t\right)} w^{1-p^{\prime}}(x)\left(d\left(x_{0}, x\right)\right)^{-\alpha p^{\prime}} d \mu<\infty .
$$

Then $T \varphi(x)$ exists $\mu$-a.e. for arbitrary $\varphi \in L_{w}^{p}(X)$.

Proof. Fix arbitrarily $\beta>0$ and let

$$
S_{\beta}=\left\{x: d\left(x_{0}, x\right) \geq \beta\right\} .
$$

Represent $\varphi$ as follows:

$$
\varphi(x)=\varphi_{1}(x)+\varphi_{2}(x)
$$

where $\varphi_{1}(x)=\varphi(x) \chi_{s_{\beta}}(x)$ and $\varphi_{2}(x)=\varphi(x)-\varphi_{1}(x)$.

For $\varphi_{2}$ we have

$$
\begin{gathered}
\int_{X}\left|\varphi_{2}(x)\right|^{p} d \mu=\frac{v(\beta)}{v(\beta)} \int_{B\left(x_{0}, \beta\right)}|\varphi(x)|^{p} d \mu \\
\leq \frac{1}{v(\beta)} \int_{B\left(x_{0}, \beta\right)}|\varphi(x)|^{p} v\left(d\left(x_{0}, x\right)\right) d \mu \leq \frac{c_{1}}{v(\beta)} \int_{B(x, \beta)}|\varphi(x)|^{p} w(x) d \mu<\infty .
\end{gathered}
$$

Consequently, $\varphi_{2} \in L^{p}(X)$ and by Theorem $\mathrm{C}$ we have $T \varphi_{2} \in L^{p}(X)$. Hence $T \varphi_{2}(x)$ exists a.e. on $X$.

Now let $x \in X$ and let $d\left(x_{0}, x\right)<\beta / 2$. If $d\left(x_{0}, y\right) \geq \beta$, then

$$
\frac{d\left(x_{0}, y\right)}{2} \geq d(x, y)
$$

Using this inequality, we obtain

$$
\begin{gathered}
\left|T \varphi_{1}(x)\right| \leq c_{2} \int_{S_{\beta}} \frac{|\varphi(y)|}{d(x, y)^{\alpha}} d \mu \leq c_{3} \int_{S_{\beta}} \frac{|\varphi(y)|}{d\left(x_{0}, y\right)^{\alpha}} d \mu \\
\leq c_{3}\left(\int_{S_{\beta}}|\varphi(y)|^{p} w(y) d \mu\right)^{1 / p}\left(\int_{S_{\beta}} w^{1-p^{\prime}}(y) d\left(x_{0}, y\right)^{-\alpha p^{\prime}} d \mu\right)^{1 / p^{\prime}}<\infty .
\end{gathered}
$$

As we may take $\beta$ arbitrarily large, we conclude that $T \varphi(x)$ exists a.e. 
Theorem 3.2. Let $a=\infty, 1<p<\infty$ and let $v$ be a positive continuous decreasing function $(0, \infty)$. Suppose that $w$ is a weight function on $X$ and the following conditions are satisfied:

(i) for almost all $x$

$$
v\left(d\left(x_{0}, x\right) / 2\right) \leq c w(x)
$$

$$
\sup _{t>0}\left(\int_{B\left(x_{0}, t\right)} v\left(d\left(x_{0}, x\right)\right) d \mu\right)^{1 / p}\left(\int_{X \backslash B\left(x_{0}, t\right)} w^{1-p^{\prime}}(x) d\left(x_{0}, x\right)^{-\alpha p^{\prime}} d \mu\right)^{1 / p^{\prime}}<\infty .
$$

Then the operator $T$ is bounded from $L_{w}^{p}(X)$ to $L_{v\left(d\left(x_{0}, \cdot\right)\right)}^{p}(X)$.

Proof. Without loss of generality we can represent $v$ as

$$
v(t)=v(+\infty)+\int_{t}^{\infty} \phi(\tau) d \tau, \quad \phi \geq 0 .
$$

Further,

$$
\begin{gathered}
\int_{X}|T f(x)|^{p} v\left(d\left(x_{0}, x\right)\right) d x \\
=v(+\infty) \int_{X}|T f(x)|^{p} d \mu+\int_{X}|T f(x)|^{p}\left(\int_{d\left(x_{0}, x\right)}^{\infty} \phi(t) d t\right) d \mu \equiv I_{1}+I_{2} .
\end{gathered}
$$

If $v(+\infty)=0$, then $I_{1}=0$. But if $v(+\infty) \neq 0$, then by virtue of the boundedness of $T$ in $L^{p}(X)$ we have

$$
I_{1} \leq c_{1} v(+\infty) \int_{X}|f(x)|^{p} d \mu \leq c_{1} \int_{X}|f(x)|^{p} v\left(d\left(x_{0}, x\right)\right) d \mu \leq c_{2} \int_{X}|f(x)|^{p} w(x) d \mu .
$$

Now we pass to $I_{2}$ :

$$
\begin{aligned}
I_{2} & =\int_{0}^{\infty} \phi(t)\left(\int_{B\left(x_{0}, t\right)}|T f(x)|^{p} d \mu\right) d t \leq c_{3} \int_{0}^{\infty} \phi(t)\left(\int_{B\left(x_{0}, t\right)}\left|T f_{t}^{(1)}(x)\right|^{p} d \mu\right) d t \\
& +c_{3} \int_{0}^{\infty} \phi(t)\left(\int_{B\left(x_{0}, t\right)}\left|T f_{t}^{(2)}(x)\right|^{p} d \mu\right) d t=I_{21}+I_{22},
\end{aligned}
$$

where $f_{t}^{(1)}=f \chi_{B\left(x_{0}, 2 t\right)}$ and $f_{t}^{(2)}=f-f_{t}^{(1)}$. Again using Theorem $\mathrm{C}$ we have

$$
\begin{aligned}
I_{21} & \leq c_{4} \int_{0}^{\infty} \phi(t)\left(\int_{B\left(x_{0}, 2 t\right)}|f(y)|^{p} d \mu\right) d t \\
& =c_{4} \int_{X}|f(y)|^{p}\left(\int_{\frac{d\left(x_{0}, x\right)}{2}}^{\infty} \phi(t) d t\right) d \mu \leq c_{5} \int_{X}|f(y)|^{p} w(y) d \mu .
\end{aligned}
$$


It remains to estimate $I_{22}$. If $x \in B\left(x_{0}, t\right)$ and $y \in X \backslash B\left(x_{0}, 2 t\right)$, then

$$
\frac{d\left(x_{0}, y\right)}{2} \leq d(x, y) \text {. }
$$

Consequently,

$$
I_{22} \leq c_{5} \int_{0}^{\infty} \phi(t)\left(\int_{B\left(x_{0}, t\right)} d \mu\right)\left(\int_{X \backslash B\left(x_{0}, 2 a_{1} t\right)}|f(y)| d\left(x_{0}, y\right)^{-\alpha} d \mu\right)^{p} d t .
$$

Moreover,

$$
\int_{0}^{s} \phi(t)\left(\int_{B\left(x_{0}, t\right)} d \mu\right) d t=\int_{B\left(x_{0}, s\right)}\left(\int_{d\left(x_{0}, x\right)}^{s} \phi(t) d t\right) d \mu \leq \int_{B\left(x_{0}, s\right)} v\left(d\left(x_{0}, x\right)\right) d \mu
$$

and due to Proposition B we finally obtain the boundedness of $T$.

Now we are going to establish weighted estimates for the operator $T$ in Lorentz spaces defined on spaces of nonhomogeneous type. Theorem $\mathrm{C}$ and the interpolation theorem imply

Proposition E. Let $1<p, q<\infty$. Then $T$ is bounded in $L^{p q}(X)$.

The following lemmas are obtained in the same way as in the homogeneous case. Instead of Theorem A we need to use Theorem C.

Lemma 3.3. Let $1<s \leq p<\infty$. Let the weight functions $w$ and $w_{1}$ satisfy the conditions:

(1) there exists an increasing function $v$ on $(0,4 a)$ such that the inequality

$$
v\left(d\left(x_{0}, x\right)\right) \leq b w(x) w_{1}(x)
$$

holds for almost all $x \in X$;

(2) for every $t, 0<t<a$, the norm

$$
\left\|\frac{1}{w(\cdot) w_{1}(\cdot)} \chi_{\left\{d\left(x_{0}, y\right) \leq t\right\}}(\cdot)\right\|_{L_{w}^{p^{\prime} s^{\prime}}(X)}
$$

is finite.

Then $\operatorname{Tg}(x)$ exists a.e. on $X$ for any $g$ satisfying the condition

$$
\left\|g(\cdot) w_{1}(\cdot)\right\|_{L_{w}^{p s}(X)}<\infty .
$$

Lemma 3.4. Let $1<s \leq p<\infty$. Suppose also that $u$ and $u_{1}$ are positive increasing functions on $\left(0,4 a_{1} a\right)$ and

$$
\left\|\frac{1}{u\left(d\left(x_{0}, \cdot\right)\right) u_{1}\left(d\left(x_{0}, \cdot\right)\right)} \chi_{\bar{B}\left(x_{0}, t\right)}(\cdot)\right\|_{L_{u\left(d\left(x_{0}, \cdot\right)\right)}^{p^{\prime} s^{\prime}}(X)}<\infty
$$

for all $t$ satisfying the condition $0<t<a$. Then for arbitrary $\varphi$ with

$$
\left\|\varphi(\cdot) u_{1}\left(d\left(x_{0}, \cdot\right)\right)\right\|_{L_{u\left(d\left(x_{0}, \cdot\right)\right)}^{p s}(X)}<\infty,
$$

$T \varphi(x)$ exists a.e. on $X$. 
The following lemmas are also true.

Lemma 3.5. Let $a=\infty, 1<s \leq p<\infty$. Suppose that for weights $w$ and $w_{1}$ the following conditions are satisfied:

(1) there exists a decreasing positive function $v$ on $(0, \infty)$ such that

$$
v\left(d\left(x_{0}, \cdot\right)\right) \leq b w(x) w_{1}^{p}(x)
$$

for almost all $x \in X$;

(2) for every $t>0$,

$$
\left\|\frac{d\left(x_{0}, \cdot\right)^{-\alpha}}{w(\cdot) w_{1}(\cdot)} \chi_{X \backslash \bar{B}\left(x_{0}, t\right)}(\cdot)\right\|_{L_{w}^{p^{\prime} s^{\prime}(X)}}<\infty .
$$

Then $T g(x)$ exists a.e. on $X$ for arbitrary $g$ satisfying $\left\|g(\cdot) w_{1}(\cdot)\right\|_{L_{w}^{p s}(X)}<\infty$.

From the previous lemmas easily follows

Lemma 3.6. Let $a=\infty, 1<s \leq p<\infty$. Suppose also that for the decreasing functions $u$ and $u_{1}$ on $(0, \infty)$ the following condition is satisfied

$$
\left\|\frac{d\left(x_{0}, \cdot\right)^{-\alpha}}{u\left(d\left(x_{0}, \cdot\right)\right) u_{1}\left(d\left(x_{0}, \cdot\right)\right)} \chi_{X \backslash \bar{B}\left(x_{0}, t\right)}(\cdot)\right\|_{L_{u\left(d\left(x_{0}, \cdot\right)\right)}^{p^{\prime} s^{\prime}}(X)}<\infty
$$

for all $t>0$. Then $T g(x)$ exists a.e. on $X$ for $g$ satisfying the condition

$$
g(\cdot) u_{1}\left(d\left(x_{0}, \cdot\right)\right) \in L_{u\left(d\left(x_{0}, \cdot\right)\right)}^{p s}(X) .
$$

Using Propositions E and C, we obtain the following result in the same way as Theorem 2.1.

Theorem 3.3. Let $1<s \leq p \leq q<\infty$, and let $w$ be a weight function on $X$. Assume that $v$ is a positive increasing continuous function on $(0,4 a)$. Suppose also that the following two conditions are satisfied:

(1) there exists a positive constant $c$ such that the inequality

$$
v\left(2 a_{1} d\left(x_{0}, x\right)\right) \leq c w(x)
$$

holds for almost every $x \in X$;

(2)

$$
\sup _{0<t<a}\left\|\left(d\left(x_{0}, \cdot\right)\right)^{-\alpha} \chi_{X \backslash \bar{B}\left(x_{0}, t\right)}\right\|_{L_{v}^{p q}(X)}\left\|\frac{1}{w(\cdot)} \chi_{\bar{B}\left(x_{0}, t\right)}(\cdot)\right\|_{L_{w}^{p^{\prime} s^{\prime}(X)}}<\infty .
$$

Then the operator $T$ is bounded from $L_{w}^{p s}(X)$ to $L_{v\left(d\left(x_{0}, \cdot\right)\right.}^{p q}(X)$.

Theorem 3.4. Let $a=\infty$, and let $1<s \leq p \leq q<\infty$; suppose that $v$ is a positive decreasing continuous function on $(0, \infty)$. Assume also that:

(i) there exists a positive constant b such that the inequality

$$
v\left(\frac{d\left(x_{0}, x\right)}{2 a_{1}}\right) \leq b w(x)
$$

is true for a.e. $x \in X$; 
(ii)

$$
\sup _{t>0}\left\|\chi_{\bar{B}\left(x_{0}, t\right)}(\cdot)\right\|_{L_{v}^{p q}(X)}\left\|\frac{\left(d\left(x_{0}, \cdot\right)\right)^{-\alpha}}{w(\cdot)} \chi_{X \backslash \bar{B}\left(x_{0}, t\right)}(\cdot)\right\|_{L_{w}^{p^{\prime} s^{\prime}}(X)}<\infty .
$$

Then $T$ acts boundedly from $L_{w}^{p s}(X)$ into $L_{v\left(d\left(x_{0}, \cdot\right)\right.}^{p q}(X)$.

Finally, we formulate the following result:

Theorem 3.5. Let $a=\infty, 1<s \leq p \leq q<\infty$. Suppose that $v, w, u_{1}$ and $u_{2}$ are weights on $X$. Assume that the following conditions are fulfilled:

(1) there exists a positive constant $b$ such that for all $t>0$

$$
\sup _{F_{t}} v^{1 / p}(x) \sup _{F_{t}} u_{2}(x) \leq b \inf _{F_{t}} w^{1 / p}(x) \inf _{F_{t}} u_{1}(x)
$$

holds, where $F_{t}=\left\{x \in X: \frac{t}{a_{1}} \leq d\left(x_{0}, x\right)<8 a_{1} t\right\}$;

$$
\sup _{t>0}\left\|u_{2}(\cdot)\left(d\left(x_{0}, \cdot\right)\right)^{-\alpha} \chi_{X \backslash \bar{B}\left(x_{0}, t\right)}(\cdot)\right\|_{L_{v}^{p q}(X)}\left\|\frac{1}{u_{1}(\cdot) w(\cdot)} \chi_{\bar{B}\left(x_{0}, t\right)}(\cdot)\right\|_{L_{w}^{p^{\prime} s^{\prime}}(X)}<\infty ;
$$

$$
\sup _{t>0}\left\|u_{2}(\cdot) \chi_{\bar{B}\left(x_{0}, t\right)}(\cdot)\right\|_{L_{v}^{p q}(X)}\left\|\left(d\left(x_{0}, \cdot\right)\right)^{-\alpha}\left(u_{1}(\cdot) w(\cdot)\right)^{-1} \chi_{X \backslash \bar{B}\left(x_{0}, t\right)}(\cdot)\right\|_{L_{w}^{p^{\prime} s^{\prime}}(X)}<\infty .
$$

Then the following inequality holds:

$$
\left\|u_{2}(\cdot) T f(\cdot)\right\|_{L_{v}^{p q}(X)} \leq c\left\|u_{1}(\cdot) f(\cdot)\right\|_{L_{w}^{p s}(X)},
$$

where the positive constant $c$ does not depend on $f$.

Remark 3.1. The results of this section remain valid if we do not require the continuity of $v$, but assume that $\mu B\left(x_{0}, r\right)$ is continuous with respect to $r$.

\section{EXAMPLES OF WEIGHT FUNCTIONS}

Let $(X, d \mu)$ be an SHT such that the condition $\mu(B(x, r)) \approx r$ holds (if $a<\infty$, then we assume that this condition is fulfilled for $0<r \leq 1$ ). It is known (see [5]) that if $\mu(X)<\infty$, then

$$
\int_{X}|K f(x)|^{p}\left(d\left(x_{0}, x\right)\right)^{p-1} d \mu \leq c \int_{X}|f(x)|^{p}\left(d\left(x_{0}, x\right)\right)^{p-1} \log ^{p} \frac{b}{d\left(x_{0}, x\right)} d \mu,
$$

where $x_{0} \in X, 1<p<\infty$, and $b=8 a_{1} a e^{p^{\prime}}$ and the positive constant $c$ does not depend on $f$.

Example 4.1. Let $1<p<q<\infty$, and let $a<\infty$; put $v(t)=t^{p-1}$, $w(t)=t^{p-1} \ln ^{\gamma} \frac{b}{t}$ for $t \in\left(0,4 a_{1} a\right)$, where $b=8 a_{1} a e^{\frac{\gamma}{p-1}}, \gamma=\frac{p}{q}+p-1$, and $a_{1}$ is the positive constant from Definition 1.1. Then from Theorem 2.1 it follows that

$$
\|K f(\cdot)\|_{L_{v\left(d\left(x_{0}, \cdot\right)\right)}^{p q}(X)} \leq c\|f(\cdot)\|_{L_{w\left(d\left(x_{0}, \cdot\right)\right)}^{p}(X)},
$$

where the positive constant $c$ does not depend on $f$. 
Example 4.2. Let $1<p<q<\infty, a=\infty, v(t)=t^{p-1}$ when $0<t \leq 1$ and $v(t)=t^{\alpha}$ when $t>1$, and let $w(t)=t^{p-1} \ln ^{\gamma} \frac{2 e^{\frac{\gamma}{p-1}}}{t}$ when $t \leq 1$, and $w(t)=t^{\beta} \ln ^{\beta}\left(2 e^{\frac{\gamma}{p-1}}\right)$ when $t>1$, where $\gamma=\frac{p}{q}+p-1,0<\alpha \leq \beta<p-1$. Then inequality (4.1) holds.

An appropriate example for the conjugate function

$$
\widetilde{f}(x)=\frac{1}{\pi} \int_{-\pi}^{\pi} \frac{f(x+t)}{2 \operatorname{tg} \frac{t}{2}} d t
$$

is presented in [11].

\section{REFERENCES}

1. H.-M. Chung, R. A. Hunt, and D. S. Kurtz, The Hardy-Littlewood maximal function on $L(p, q)$ spaces with weights. Indiana Univ. Math. J. 31(1982), 109-120.

2. R. R. Coifman and G. Weiss, Extensions of Hardy spaces and their use in analysis. Bull. Amer. Soc. 83(1977), 569-645.

3. D. E. Edmunds and V. Kokilashvili, Two-weight inequalities for singular integrals. Canadian Math. Bull. 38(1995), 119-125.

4. D. E. Edmunds, V. M. Kokilashvili, and A. Meskhi, Boundedness and compactness of Hardy-type operators on Banach function spaces defined on measure space. Proc. A. Razmadze Math. Inst. 117(1998), 7-30.

5. D. E. Edmunds, V. Kokilashvili, and A. Meskhi, Two weight estimates for singular integrals defined on spaces of homogeneous type. Canadian J. Math. 52(2000), No. 3, 468-502.

6. I. Genebashvili, A. Gogatishvili, V. Kokilashvili, and M. Krbec, Weight theory for integral transform on spaces of homogeneous type. Pitman Monographs and Surveys in Pure and Applied Mathematics, Longman, Harlow, 1998.

7. V. Guliev, Two-weight $L_{p}$ inequality for singular integral operator on Heisenberg groups. Georgian Math. J. 1(1994), No. 4, 367-376.

8. E. G. Gusseinov, Singular integrals in the space of function summable with monotone weight. (Russian) Mat. Sb. 132(174)(1977), No. 1, 28-44.

9. S. Hoffman, Weighted norm inequalities and vector-valued inequalities for certain rough operators. Indiana Univ. Math. J. 42(1993), 1-14.

10. R. A. Hunt, on $L(p, q)$ spaces. L'enseign. Math. 12(1966), 249-276.

11. V. Kokilashvili, On two-weight inequalities for conjugate functions. Proc. A. Razmadze Math. Inst. 112(1997), 141-142.

12. V. Kokilashvili and M. Krbec, Weighted inequalities in Lorentz and Orlicz spaces. World Scientific, Singapore, New Jersey, London, Hong Kong, 1991.

13. V. Kokilashvili and A. Meskhi, Two-weight inequalities for singular integrals defined on homogeneous groups. Proc. A. Razmadze Math. Inst. 112(1997), 57-90.

14. V. Kokilashvili and A. Meskhi, Two-weight inequalities for Hardy type transforms and singular integrals defined on homogeneous type spaces. Proc A. Razmadze Math. Inst. 114(1997), 119-123. 
15. V. Kokilashvili and A. Meskhi, Two-weight estimates for singular integrals on homogeneous groups. (Russian) Doklady Akademii Nauk 354(1997), No. 3, 301-303. English transl. in Doklady Mathematics 55(1997), 362-364.

16. V. Kokilashvili and A. Meskhi, Two-weight inequalities for integral operators in Lorentz spaces defined on homogeneous groups. Georgian Math. J. 6(1999), No. 1, 65-82.

17. B. Muckenhoupt and R. L. Wheeden, Two-weight function norm inequalities for the Hardy-Littlewood maximal function and the Hilbert transform. Studia Math. 55(1976), No. 3, 279-294.

18. F. Nazarov, S. Treil, and A. Volberg, Weak type estimates and Cotlar inequalities for Calderón-Zygmund operators on nonhomogeneous spaces. International Mathematics Research Notices 9(1998), 463-487.

19. Y. Rakotondratsimba, On boundedness of classical operators on weighted Lorentz spaces. Georgian Math. J. 5(1998), No. 2, 177-200.

20. E. T. SAWyer and R. L. Wheeden, Weighted inequalities for fractional integrals on Euclidean and homogeneous spaces. Amer. J. Math. 114(1992), 813-874.

21. E. M. Stein, Harmonic analysis: real variable methods, orthogonality and oscillatory integrals. Princeton University Press, Princeton, New Jersey, 1993.

22. I. M. Stein and G. WeIss, Introduction to Fourier analysis on Euclidean spaces. Princeton, New Jersey, Princeton University Press, 1971.

23. J. O. Strömberg and A. Torchinsky, Weighted Hardy spaces. Lecture Notes in Math. 1381, Springer Verlag, Berlin, 1989.

(Received 23.08.2000)

Authors' Addresses:

D. E. Edmunds

Centre for Mathematical Analysis

and its Applications

University of Sussex

Brighton BN1 9QH

Sussex

United Kingdom

E-mail: D.E.Edmunds@sussex.ac.uk

V. Kokilashvili and A. Meskhi

A. Razmadze Mathematical Institute

Georgian Academy of Sciences

1, M. Aleksidze St., Tbilisi 380093

Georgia

E-mail: kokil@rmi.acnet.ge

meskhi@rmi.acnet.ge 